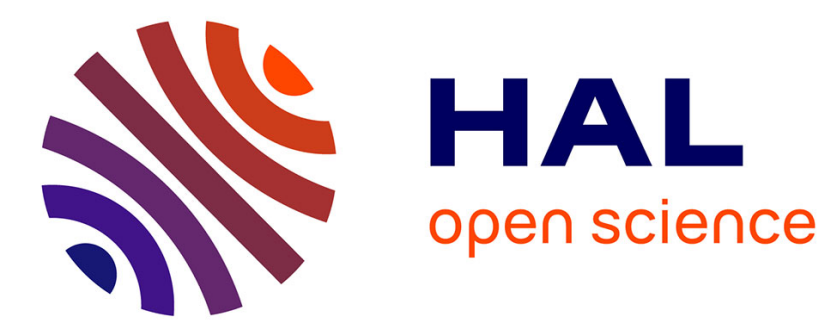

\title{
Continuous monitoring of a surface slope by real-time shearing interferometry with a photorefractive crystal.
}

\author{
Gilles Pauliat, Gérald Roosen
}

\section{To cite this version:}

Gilles Pauliat, Gérald Roosen. Continuous monitoring of a surface slope by real-time shearing interferometry with a photorefractive crystal.. Applied optics, 2006, 45 (5), pp.993-999. hal-00856108

\section{HAL Id: hal-00856108 \\ https://hal-iogs.archives-ouvertes.fr/hal-00856108}

Submitted on 30 Aug 2013

HAL is a multi-disciplinary open access archive for the deposit and dissemination of scientific research documents, whether they are published or not. The documents may come from teaching and research institutions in France or abroad, or from public or private research centers.
L'archive ouverte pluridisciplinaire HAL, est destinée au dépôt et à la diffusion de documents scientifiques de niveau recherche, publiés ou non, émanant des établissements d'enseignement et de recherche français ou étrangers, des laboratoires publics ou privés. 


\title{
Continuous monitoring of a surface slope by real-time shearing interferometry with a photorefractive crystal
}

\author{
Gilles Pauliat and Gérald Roosen
}

\begin{abstract}
We propose and demonstrate a new technique for measuring the temporal variations of the surface slope of an object. This real-time shearometric arrangement takes advantage of the dynamic properties of holograms in photorefractive crystals. The accuracy of the measurements should make this technique suitable for real-time structural intensity determinations. () 2006 Optical Society of America

OCIS codes: $120.2880,160.5320,190.5330,120.7280$.
\end{abstract}

\section{Introduction}

Photorefractive materials are attractive recyclable holographic media for holographic interferometry. ${ }^{1-10}$ The holograms automatically self-develop in these indefinitely reusable materials. Most of the experiments are conducted in a real-time configuration, which is one of the simplest configurations. First a hologram of the object is recorded by the interference of the object beam with a reference beam. Subsequently, after the object has moved or during this movement, the object beam interferes with the beam reconstructed by diffraction from the reference beam and is recorded by a CCD camera. These two wavefronts represent the current state of the object and the object in its previous state, respectively, as it was during hologram recording. The interferograms are thus grabbed by the CCD camera in real time, and their analyses give information about the object deformations. This real-time configuration allows monitoring of the object movement by taking a succession of interferograms with the same reference hologram.

Although photorefractive crystals are less sensitive than silver halide plates, their resolution is also very high: The minimum fringe spacing that can be effi-

\footnotetext{
The authors are with the Laboratoire Charles Fabry de l'Institut d'Optique, du Centre National de la Recherche Scientifique et de l'Université Paris-Sud, Bâtiment 503, Centre Scientifique d'Orsay, F-91403 Orsay Cedex, France. G. Pauliat's e-mail address is gilles.pauliat@iota.u-psud.fr.

Received 21 April 2005; revised 29 July 2005; accepted 29 July 2005.

0003-6935/06/050993-07\$15.00/0

(C) 2006 Optical Society of America
}

ciently recorded in photorefractive crystals is usually of the order of a few hundred nanometers. The resolution of the hologram is thus limited only by the optical lenses but not by the photorefractive material. This very high resolution leads to high-quality interferograms. ${ }^{8-10}$ The analyses of these interferograms thus produce more-accurate measurements than those obtained with speckle interferometry ${ }^{6}$ : Measurement errors smaller than $\lambda / 40 \mathrm{rms}$ are obtained on each pixel of the CCD camera whose size is typically $512 \times 512$ pixels. ${ }^{10}$

This quality, combined with the simplicity of the experimental setup, makes photorefractive crystals good candidates for replacing silver halide plates. In spite of these obvious advantages, interferometry with photorefractive materials usually suffers from the same drawbacks as classical holographic interferometry:

- Unless expensive pulsed lasers are used, the hologram must be recorded with the object at rest with vibration isolation equipment.

- If the laser is not fixed to the optical setup, the reference beam must be readjusted each time the optical setup is moved.

- The laser coherence length must be larger than the maximum optical path difference between the reference and the object beams. This optical path is imposed by the field of view and the object depth. Single-mode lasers are thus required.

Shearography was proposed for relaxing some of these constraints: It does not require large coherence lengths and the absence of a reference beam avoids any need for alignment before the measurements. ${ }^{11-13}$ 


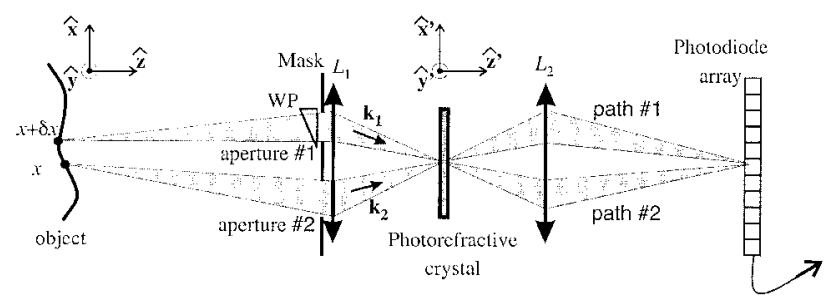

Fig. 1. Principle of a real-time dynamic shearing interferometer. For the sake of clarity, only the interfering beams for two points are represented, and the laser beam illuminating the object is not shown. WP, wedged plate.

However, shearographic measurements are usually less accurate than holographic measurements.

In this paper we propose an alternative solution that combines some advantages of real-time photorefractive holographic interferometry with those of shearography. With this new technique we can continuously monitor the gradient of the normal displacement of an object. The self-referenced hologram is recorded while the object moves, and the absence of any reference beam makes this system relatively insensitive to environmental noise.

This technique could be the basis of a device aimed at measuring the structural intensity in plates. ${ }^{14-16}$ Indeed structural intensity calculations require knowledge of the first and the second spatial derivatives of the normal displacement. As described below, this structural intensity could be computed from the simultaneous measurements of the gradient displacements on some adjacent points by the same probe.

After a brief presentation of the proposed principle, we detail the experimental setup that we developed to validate this principle. Advantages, limitations, and further possible improvements are then discussed.

\section{Principle: Image Shearing for Real-Time Interferometry}

The principle of a real-time shearing interferometer is depicted by the diagram shown in Fig. 1. Two shifted images of the object are the first projected into the photorefractive crystal. From among the conventional shearing systems ${ }^{11,12}$ (such as Michelson interferometers, split lenses, wedge plates, Wollaston prisms, and diffraction gratings), we use a mask in front of the first lens $L_{1}$ to define two beam paths. Lens $L_{1}$ thus forms two overlapping images of the object at its image plane set in the middle of the photorefractive crystal. The wedge plate shifts one of these images in a direction defined by the wedge orientation. In Fig. 1, and hereafter, this shift is along the $\hat{\mathbf{x}}$ direction. The spacing between the two well-separated apertures defines an average fringe carrier for recording a hologram between the two images; this fringe carrier is within the sensitivity domain of the photorefractive crystal.

At crystal location $x^{\prime}$, the image of point $x$ by aperture 1 interferes with the image of point $x+\delta x$ by aperture 2 . In complex notations, and on the crystal, the electric fields of these two interfering optical waves are expressed by

$$
\begin{aligned}
E_{1}(x)= & A_{1}(x) \exp [i \varphi(x, t)] \exp \left(i \mathbf{k}_{1} \cdot \mathbf{r}\right), \\
E_{2}(x+\delta x)= & A_{2}(x+\delta x) \exp [i \varphi(x+\delta x, t)] \\
& \times \exp \left(i \mathbf{k}_{2} \cdot \mathbf{r}\right),
\end{aligned}
$$

where $A_{1,2}$ are the complex amplitudes, $\mathbf{k}_{1,2}$ are the average wave vectors (see Fig. 1), $\mathbf{r}$ is the space vector, and $t$ is the time.

Amplitudes $A_{1,2}$ include the time-averaged phase of the beams. $\varphi(x, t)$ and $\varphi(x+\delta x, t)$ are the temporal phase shifts induced by object vibration. If the object is illuminated by a beam at normal incidence, then ${ }^{11}$

$$
\varphi(x, t)=\frac{4 \pi}{\lambda} w(x, t),
$$

where $w(x, t)$ is the surface displacement along the $z$ axis of the point at the $x$ coordinate.

With shearography, we intend to measure

$$
\Delta \varphi(x, t)=\varphi(x+\delta x, t)-\varphi(x, t) \approx \frac{4 \pi}{\lambda} \frac{\partial w(x, t)}{\partial x} \delta x
$$

where $\partial w / \partial x$ is the change in surface slope induced by the vibration.

The two incoming beams record a hologram. A photorefractive crystal used in the diffusion regime (i.e., without any applied electric field and without any photovoltaic effect) is less sensitive to the low-spatialfrequency intensity speckle patterns, ${ }^{17}$ such as the speckle patterns of each beam, and we thus may neglect these spatial components and take into account only the hologram resulting from the interference between the two beams whose wave vector is about $\mathbf{k}_{H}=k_{H} \hat{\mathbf{x}}^{\prime}=\mathbf{k}_{1}-\mathbf{k}_{2}$.

The hologram buildup time in photorefractive crystals is inversely proportional to the intensity. By adjustment of this intensity, it is set longer than the largest period of the vibration. The photorefractive crystal is then sensitive to the time average of the modulation ratio of the interference pattern. ${ }^{17}$ Thus, when the photorefractive crystal thickness is neglected, the amplitude of the refractive index hologram is

$$
\delta n=\xi C \frac{A_{1}(x) A_{2}^{*}(x+\delta x)}{\left|A_{1}\right|^{2}+\left|A_{2}\right|^{2}} \exp \left[i\left(k_{H} x^{\prime}+\psi\right)\right]
$$

where

$$
C \approx \exp (-t / \tau) \int_{0}^{t} \exp \left[i \Delta \varphi\left(x, t^{\prime}\right)\right] \exp \left(t^{\prime} / \tau\right) \frac{\mathrm{d} t^{\prime}}{\tau},
$$

and $\psi$ is a phase shift specific to the photorefractive effect. It is equal to $\psi= \pm \pi / 2$ for a photorefractive 


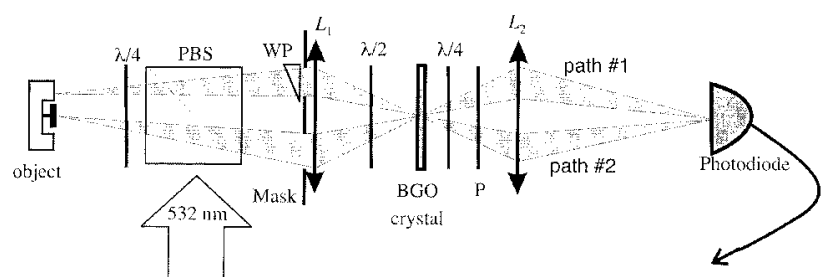

Fig. 2. Experimental setup used for the first demonstration. PBS, polarizing beam splitter; $\mathrm{BGO}, \mathrm{Bi}_{12} \mathrm{GeO}_{20} ; \mathrm{P}$, polarizer.

crystal used in the diffusion regime. ${ }^{17}$ The proportionality coefficient $\xi$ in Eq. (2) is real.

$C$ represents the decrease in the hologram strength at time $t$, which is due to the blurring of the interference pattern over the built-up time constant $\tau$ of the photorefractive crystal. ${ }^{18,19}$ To keep the refractive index modulation at the maximum, the amplitude of the vibration, governed by $\Delta \varphi(x, t)$, should be much smaller than $\pi$ so as not to blur the hologram. $C$ is thus equal to 1 for $t \gg \tau$.

In case a slow disturbance of the interference pattern appears (caused by, for instance, air flows), the hologram adapts itself to the new interference pattern.

In this system, the hologram reconstruction is performed in real time by the two recording beams. Each of them is diffracted onto the stationary hologram and produces a wavefront, which is superimposed on the transmitted wavefront of the other beam. The transverse structure of the diffracted wavefront reproduces the transmitted wavefront. However, these wavefronts differ in phase by $\Delta \varphi(x, t)$. They are made to interfere onto a photodetector array in an image plane of the object. The beating signal makes it possible to monitor $\Delta \varphi(x, t)$. With a photodetector array, several measurements on a series of pairs of points can be performed simultaneously. The exact dependence of the detected electric signal on $\Delta \varphi(x, t)$ varies as a function of (i) the polarizing properties of the photorefractive crystal, (ii) the optical beam polarizations, (iii) the value of $\psi$, and (iv) the possible presence and orientation of wave plates and polarizers.

In the experimental validation of this technique presented below, we investigated one possible scheme to recombine the diffracted beam with the transmitted beam.

\section{Experimental Setup}

We validated this principle with the experimental setup shown in Fig. 2. For this first demonstration we compared only the relative movement of two points by using a single-element detector. The laser is a cw frequency-doubled Nd:YAG laser at $532 \mathrm{~nm}$ whose power is $140 \mathrm{~mW}$. Its beam is first shaped by a cylindrical lens and sent onto the object by means of the polarizing beam-splitter cube. The shape of the elliptical illuminated area is approximately $4 \mathrm{~mm} \times 15 \mathrm{~mm}$ with an optical power of $60 \mathrm{~mW}$. The quarter-wave plate in front of the beam-splitter cube makes the optical polarization circular on the object. If the light

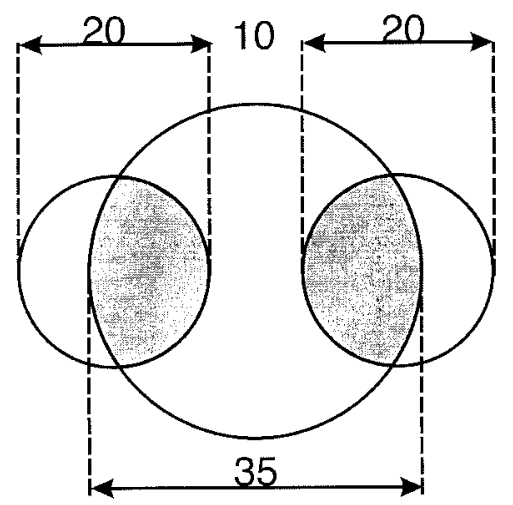

Fig. 3. Sizes of the apertures in millimeters; the apertures are shaded.

is not fully depolarized by the diffuse reflection, this arrangement makes the polarization of the scattered light, sent back through the quarter-wave plate, perpendicular to the linear incident-light polarization. The scattered light thus goes through the polarizing beam-splitter cube with minimum losses.

First, lens $L_{1}$ images the object onto the photorefractive crystal with a transverse magnification equal to $\gamma=1 / 3$. The focal length of this plano-convex lens is $100 \mathrm{~mm}$; the diameter of its mount is $35 \mathrm{~mm}$. Two circular holes, which are $20 \mathrm{~mm}$ in diameter, are drilled into the metallic plate set in front of this lens. The lens holder and this plate define a mask with two apertures whose areas are shaded in Fig. 3. On one of these apertures, the wedge plate deflects the beam by $1^{\circ}$. This corresponds to an image shift (measured in the object plane) of $7 \mathrm{~mm}$. The centers of the apertures are spaced at $\sim 20 \mathrm{~mm}$ to make the average wave vector $\mathbf{k}_{H}$ large enough to record a hologram with a significant diffraction efficiency in the photorefractive crystal. This is a copper-doped $\mathrm{Bi}_{12} \mathrm{GeO}_{20}$ crystal grown by J. C. Launay at the Université de Bordeaux in France. ${ }^{20}$ Its input face is $10 \mathrm{~mm} \times$ $10 \mathrm{~mm}$ and its thickness is $l=2.69 \mathrm{~mm}$ along the crystallographic [1힐 direction. Because of optical activity, the polarization plane of the incident light rotates by $\rho l$ while passing through the crystal, where $\rho$ is the optical activity; $\rho \approx 32.5^{\circ}$ at $532 \mathrm{~nm}$. We chose to use this crystal in the so-called anisotropic diffraction configuration. ${ }^{3,4}$ Other configurations could have been used. Nevertheless, in the anisotropic configuration, if the incident polarization is set at an angle of $\rho l / 2$ to the [110] crystallographic axis, then the transmitted and diffracted beams are orthogonally polarized. The half-wave plate in front of the crystal adjusts this polarization for this configuration. This peculiarity allows the relative average phase between these two beams to be controled by means of other wave plates located after the crystal.

Just after the photorefractive crystal, and before any wave plates, the relative phase between the transmitted wave and the diffracted wave depends on the photorefractive effect through the phase $\psi= \pm \pi / 2$ [Eq. (4)]. Along path 1 , the electric field $E^{\prime}(x)$ of the beam from 
the object point at $x$ interferes with the electric field $E^{\prime}(x+\delta x)$ of the light diffracted by the induced grating and originating from an object point at $x+\delta x$. Taking the low diffraction efficiency into account, we obtain from Eqs. (1) and (4)

$$
\begin{aligned}
E^{\prime}(x) & =A_{1}(x) \exp \left\{i\left[\mathbf{k}_{1} \cdot \mathbf{r}+\varphi(x, t)\right]\right\} \\
E^{d}(x+\delta x) & =\beta C A_{1}(x) \exp \left\{i\left[\mathbf{k}_{1} \cdot \mathbf{r}+\varphi(x+\delta x, t)\right]\right\} .
\end{aligned}
$$

Because diffraction on an index grating shifts the diffracted light by $\pi / 2$ and because the photorefractive phase shift $\psi$ equals $\pm \pi / 2$, coefficient $\beta$ is real, positive, or negative according to the orientation of the photorefractive crystal. The exact expression for $\beta$ is not trivial, first because of the speckled nature of the optical field and second because the index variation given by Eq. (4) is valid in an image plane only and not in the whole crystal thickness, which is not negligible compared with the depth of focus of the system. To determine the exact expression for $\beta$ one would need to take into account the expression of the index gratings outside the image plane and to perform the summation of the light diffracted by all these layers. In the following discussion we do not need this exact expression and we rely on approximate expressions (5).

Using notations similar to the ones used for path 1 , we have in path 2

$$
\begin{aligned}
E^{\prime}(x+\delta x) & =A_{2}(x+\delta x) \exp \left\{i\left[\mathbf{k}_{2} \cdot \mathbf{r}+\varphi(x+\delta x, t)\right]\right\} \\
E^{d}(x) & =\beta^{\prime} C A_{2}(x+\delta x) \exp \left\{i\left[\mathbf{k}_{2} \cdot \mathbf{r}+\varphi(x, t)\right]\right\} .
\end{aligned}
$$

Projecting the beams, in either path 1 or 2 , onto a polarizer would provide a beating signal in cosine:

$$
\cos [\Delta \varphi(x, t)]
$$

Because $\Delta \varphi$ is much smaller than $\pi / 2$, a cosine signal such as the one given by expression (7) is not suitable for analysis.

To make this beating signal more suitable, we insert a quarter-wave plate on the two beam paths oriented so that it induces an additional $\pi / 2$ phase shift between the orthogonally polarized transmitted and diffracted beams (Fig. 2). These two polarizations are then projected onto the polarizer axis. Angle $\Theta$ between the polarizer axis and the transmitted light polarization is experimentally adjusted for the best signal-to-noise ratio. Lens $L_{2}$ (identical to lens $L_{1}$ ) recombines the beams on the photodiode set in an image plane. We detect

$$
\begin{aligned}
I(x, \delta x)= & \left|A_{1}(x)\right|^{2}\left\{\cos ^{2} \Theta+\beta^{2} C^{2} \sin ^{2} \Theta\right. \\
& +2 \beta C \sin \Theta \cos \Theta \sin [\Delta \varphi(x, t)]\} \\
& +\left|A_{2}(x+\delta x)\right|^{2}\left\{\cos ^{2} \Theta+\beta^{\prime 2} C^{2} \sin ^{2} \Theta\right. \\
& \left.+2 \beta^{\prime} C \sin \Theta \cos \Theta \sin [\Delta \varphi(x, t)]\right\} .
\end{aligned}
$$

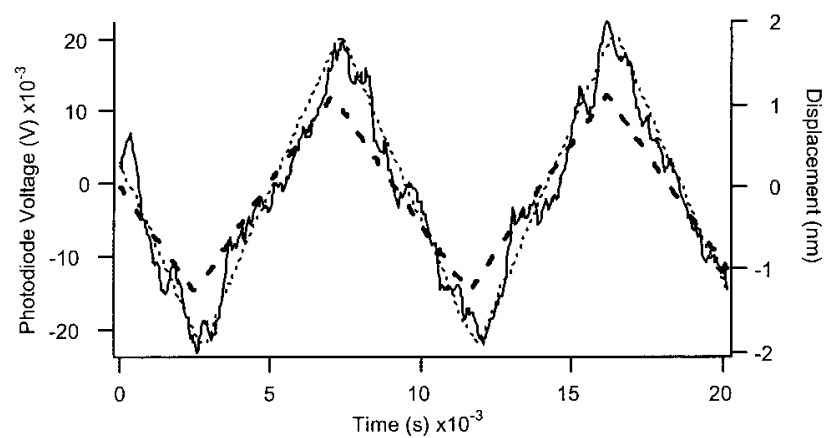

Fig. 4. The ac part of the signal detected by the photodiode (solid curve, before averaging; dotted curve, after averaging) compared with the sawtooth displacement of the piezoelectric transducer (thick dashed curve).

As shown in Eq. (8), in this optical arrangement the two paths contribute to the same beating signal on the photodiode.

The diffraction efficiency in the $\mathrm{Bi}_{12} \mathrm{GeO}_{20}$ sample we are using is quite low, of the order of $0.1 \%$, which corresponds to $\beta \approx \beta^{\prime} \approx 3 \%$. The signal of interest being given by the ac part of Eq. (8), it is usually relatively small compared with the dc component. We thus simplify Eq. (8) as follows:

$$
I(x, \delta x)=I_{0}\{1+m C \sin [\Delta \varphi(x, t)]\} .
$$

The expressions for $I_{0}$ and $m$ are derived from Eq. (8).

\section{Results}

We tested this setup by studying an object with known displacements: a metallic disk glued onto a piezoelectric transducer; this vibrating disk fits into a hole drilled inside the fixed housing. The first point under study is on the vibrating disk, whereas the other lies on the fixed housing. We tested the setup with either the bare metallic surface or by using a fully depolarizing and scattering dull white coating (correction fluid). We did not observe any noticeable change in the measurement except for the total amount of light collected by the optics and thus for the hologram buildup time. The results reported here were obtained with the white coating.

The total light power onto the crystal was $17 \mu \mathrm{W}$. This corresponds to a measured photorefractive time constant of $\tau=250 \mathrm{~ms}$. Although the photodiode frequency cutoff was $2 \mathrm{kHz}$, the experiments reported here were performed at $110 \mathrm{~Hz}$. Indeed, for this frequency, the disk displacement is proportional to the voltage applied to the piezoelectric transducer. This $110 \mathrm{~Hz}$ frequency thus simplifies the analyses. We also checked that it remains larger than the inverse of the photorefractive time constant, as discussed above. In Fig. 4, we plotted the ac part of the photodiode signal versus time and we compared this curve with the known sawtooth displacement of the piezoelectric transducer. The dc part was equal to $13 \mathrm{~V}$. For these small displacements, the signal was corrupted by the electronic amplifier noise. This noise 


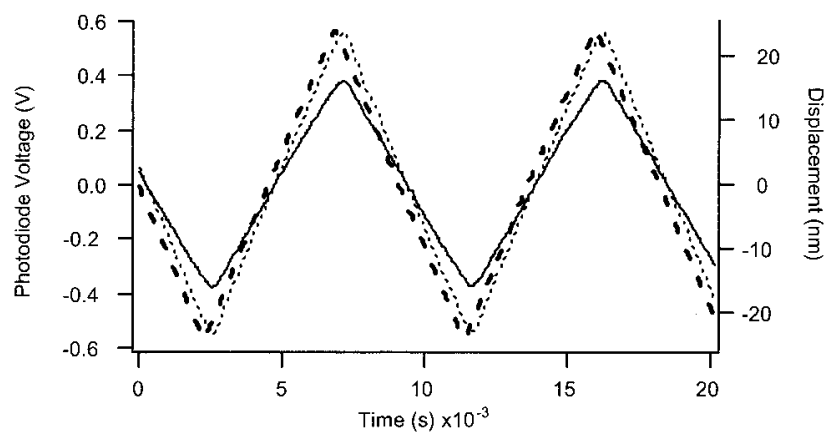

Fig. 5. Comparison of the displacement (dotted curve) computed from the signal detected by the photodiode (solid curve) with the known sawtooth displacement of the piezoelectric transducer (thick dashed curve).

was eliminated by averaging of the signal. The photodiode signal exactly reproduces the displacement because the sine in Eq. (9) can be approximated by its argument. The small temporal shift visible in Fig. 4 between the known displacement and the photodiode voltage is an electronic artifact. Because of the very small displacements involved here, coefficient $C$ is equal to unity. This allows us to calibrate the system, that is, to determine the value of $m$ in Eq. (9). For true measurements (i.e., not on a calibrated transducer) the linearity coefficient between the measured voltage and the displacement must also be calibrated. Indeed, the amplitude of detected signal depends on many factors, such as the object reflectivity. This calibration should thus be performed for each object and each of its points. One possibility would be to set a liquid-crystal phase shifter on one of the two apertures. During a calibration step, which may last for a second or so, a known phase shift is introduced by the liquid crystal. It produces a photodiode signal whose amplitude is used to calibrate the system. This calibration system was not implemented in this first demonstration.

For larger displacements, the displacement and the photodiode signal are no longer proportional, first because of the sine dependence in Eq. (9) and second because of coefficient $C$, which decays and is a function of the unknown object displacement. Nevertheless, relatively good values for the displacements can be derived from the measured signals if $\Delta \varphi(x, t)$ remains smaller than $\sim \pi / 2$. For instance, in Fig. 5 we have plotted as a solid curve the signal detected by the photodiode; this is the raw signal from the photodiode without any averaging or filtering. From this signal we obtain a first estimation of the displacement, taking into account the previous system calibration, assuming $C \approx 1$, and inverting the sine in Eq. (9). This crude estimation of the displacement allows us to compute more precisely $C$ [from Eq. (4)] and thus to refine our estimation of the displacement. If needed, this procedure can be reiterated. In Fig. 5 we have plotted as a dotted curve the displacement computed in this way. One sees that this procedure gives quite a good estimation for the true displace- ment. For larger displacements, the error increases. For example, we got an error of $30 \%$ for sawtooth displacements of $\pm 50 \mathrm{~nm}$. Nevertheless, for even larger displacements, this procedure leads to inconsistent results because several temporal displacements can lead to similar signals.

\section{Limitations}

Besides its obvious advantages (the continuous monitoring of the surface slope), this real-time shearing interferometer also presents the following specific limitations.

\section{A. Signal Corruption by In-Plane Displacement}

If there is an in-plane displacement of the object along the $x$ or $y$ direction, the speckle pattern moves along the same direction onto the crystal. This movement in the stationary index grating induces polarization changes and energy redistribution among the beams. ${ }^{21,22}$ This produces signal variations on the photodiode, which corrupts the desired out-of-plane signal. Because of the fringe modulation along the $x$ axis, an in-plane displacement along this axis produces much larger changes than along the $y$ direction. The $x$-axis displacement and the out-of-plane movement result from diffraction on the same index grating.

In our setup we experimentally found that a given in-plane displacement along the $x$ axis produces a signal equal to $\sim 0.4 \%$ of the signal produced by the same relative displacement between the two points along the $z$ axis. This parasitic signal is low because, conversely to the system described in Ref. 22, we detect the whole beam, thus integrating the signal over a large number of speckle dots. With the inplane displacement producing a signal inversely proportional to the transverse magnification $\gamma$, we expect this parasitic signal to be fully negligible for systems with smaller transverse magnifications, i.e., with systems optimized for observing large areas.

\section{B. Spatial Resolution Limited by the Photodiodes}

The photodiodes integrate the signals over their whole surfaces. In our setup, the photodiodes extend along the $x$ axis over $1.1 \mathrm{~mm}$. Because the transverse magnification between the object and the photodiode is unity, this limits the spatial resolution to the same value. This resolution could easily be increased by a reduction in the photodiode size, at the expense of the optical energy collected.

\section{Spatial Resolution Limited by the Crystal Thickness}

Crystal thickness also deteriorates the spatial resolution. Indeed, ray fan $x 1$, from point $x$ and limited by aperture 1 , intersects the ray fan from point $x+\delta x$ limited by aperture 2 in the image plane set in the middle of the crystal. Because of the crystal thickness, outside the image plane, ray fan $x 1$ also intersects rays issued from points in the vicinity of $x$ $+\delta x$. The extension of this vicinity depends on the aperture of each ray fan (given by apertures 1 and 2), on the angle between the two paths in the crystal (the 
refractive index at $\lambda=532 \mathrm{~nm}$ is equal to 2.6), and on the crystal thickness.

We measured this resolution by translating the object along the $x$ direction, starting with one point on the vibrating disk and the other on the fixed housing and finishing with the two points on this fixed housing. The electric-signal magnitude changes from $90 \%$ to $10 \%$ for a translation of approximately $2 \mathrm{~mm}$. This value corresponds to the loss of resolution induced by the crystal thickness and the photodiode size as described in the above paragraph.

One could increase the resolution by different techniques, for example, by using a thinner photorefractive sample. To keep the same diffraction efficiency, photorefractive crystals with larger nonlinearities have to be used. One could also decrease the angle between the two paths. In a $\mathrm{Bi}_{12} \mathrm{GeO}_{20}$ crystal, decreasing this angle also diminishes the diffraction efficiency unless an external electric field is applied to the sample.

\section{Cutoff Frequency}

In our first demonstration, we have been able to monitor vibration frequencies up to a few kilohertz, this frequency being limited by the piezoelectric transducer frequency cutoff. However, the signal results from diffraction on a stationary grating, which is a fully passive process. The upper cutoff frequency is thus imposed by the photodiodes and by the optical power. If enough optical power is available, such realtime photorefractive interferometers are able to monitor vibrations at frequencies larger than a few megahertz. ${ }^{9}$

\section{Further Improvements}

The dynamic range of the measurements is limited by the amplifier noise that amplifies both the useless strong dc component and the desired ac component. This useless dc component could be eliminated by use of a twin photodiode balanced arrangement. Indeed, as seen in Eq. (8), the sign of the ac signal is determined by the polarizer orientation (angle $\Theta$ ). Using a polarizing beam splitter, one can arrange the polarizations so that the two photodiodes detect two opposite ac signals superimposed on two identical dc components. Mounting these photodiodes in a light balance detection circuit subtracts the two signals and thus detects the ac part only. With this detection scheme, laser intensity noise, which appears with the same sign on the two signals, is also eliminated.

Instead of measuring signals with opposite phases with the two photodiodes, one could also play with the optical polarizations to detect a first signal proportional to $\cos [\Delta \varphi(x, t)]$ and the other proportional to $\sin [\Delta \varphi(x, t)]$ with an arrangement similar to the one discussed in Ref. 7. In such a case the system calibration may be avoided and slightly larger relative displacements could be measured.

The same principle can be extended to perform simultaneous measurements on a series of pairs of points. For instance, a straightforward extension could be obtained if the single-element photodiode used here is replaced with a linear photodiode array aligned along the $x$ axis. With such an array one could even hope to be able to measure absolute displacements or, equivalently, the displacement to each point of the line relative to a reference point on the border of the line.

Another interesting possibility would be to construct a probe to perform simultaneous measurements on five points, a central point plus four points arranged crosswise. Such a probe would directly allow the measurements of the first and second derivatives of the normal displacement $(\partial w / \partial x, \partial w / \partial y$, $\partial^{2} w / \partial x^{2}, \partial^{2} w / \partial y^{2}$, and $\left.\partial^{2} w / \partial x \partial y\right)$ along the two orthogonal directions. Such a device would allow the direct measurement of the structural intensity.

\section{Conclusion}

Combining some of the advantages of real-time holographic interferometry in a photorefractive crystal with those of shearography, we have proposed a new technique to temporally follow the changes in object surface slopes. Our first demonstration validated this technique: The hologram recording is performed continuously during the measurements and while the object moves. With this setup, we measured vibrations with amplitudes up to \pm 50 $\mathrm{nm}$.

The quality of the detected signals makes us confident of the extension of this first demonstration to the simultaneous measurement of a full series of points on larger objects and also to direct computation in real time of the structural intensity of vibrating plates, even at high frequencies.

The authors are grateful to Lionel Vasserot for his participation in the experimental work.

\section{References}

1. J. P. Huignard and J. P. Herriau, "Real-time double-exposure interferometry with $\mathrm{Bi}_{12} \mathrm{GeO}_{20}$ crystals in transverse electrooptic configuration,” Appl. Opt. 16, 1807-1809 (1977).

2. T. Sato, T. Hatsusawa, and O. Ikeda, "Dynamic interferometric observation of differential movement," Appl. Opt. 22, 38953897 (1983).

3. A. A. Kamshilin and M. P. Petrov, "Continuous reconstruction of holographic interferograms through anisotropic diffraction in photorefractive crystals," Opt. Commun. 53, 23-26 (1985).

4. A. A. Kamshilin, E. V. Mokrushina, and M. P. Petrov, "Adaptive holographic interferometers operating through selfdiffraction of recording beams in photorefractive crystals," Opt. Eng. 28, 580-585 (1989).

5. R. Magnusson, X. Wang, A. Hafiz, T. D. Black, L. N. Tello, A. Haji-Sheikh, S. Konecni, and D. Wilson, "Experiments with photorefractive crystals for holographic interferometry," Opt. Eng. 33, 596-607 (1994).

6. H. Rohleder, P. M. Petersen, and A. Marrakchi, "Quantitative measurement of the vibrational amplitude and phase in photorefractive time-average interferometry: a comparison with electronic speckle pattern interferometry," J. Appl. Phys. 76, 81-84 (1995).

7. L. Labrunie, G. Pauliat, J. C. Launay, S. Leidenbach, and G. Roosen, "Real-time double exposure holographic phase shifting 
interferometer using a photorefractive crystal," Opt. Commun. 140, 119-127 (1997).

8. F. Rickermann, S. Riehemann, and G. von Bally, "Utilization of photorefractive crystals for holographic double-exposure interferometry with nanosecond laser pulses," Opt. Commun. 155, 91-98 (1998).

9. Ph. Delaye, A. Blouin, D. Drolet, L. A. de Montmorillon, G. Roosen, and J. P. Monchalin, "Detection of ultrasonic motion of a scattering surface using photorefractive InP:Fe under an applied dc field," J. Opt. Soc. Am. B 14, 1723-1733 (1997).

10. M. P. Georges and P. C. Lemaire, "Real-time stroboscopic holographic enhancement using sillenite crystals for the quantitative analysis of vibrations," Opt. Commun. 145, 249-247 (1998).

11. J. A. Leendertz and J. N. Butters, "An image-shearing specklepattern interferometer for measuring bending moments," J. Phys. E: Sci. Instrum. 3, 1107-1110 (1973).

12. Y. Y. Hung, "Shearography: a new optical method for strain measurement and nondestructive testing," Opt. Eng. 21, 391395 (1982).

13. N. K. Mohan and P. Rastogi, "Recent development in digital speckle pattern interferometry," Opt. Lasers Eng. 40, 339-444 (2003).

14. D. U. Noiseux, "Measurement of power flow in uniform beams and plates," J. Acoust. Soc. Am. 47, 238-247 (1970).

15. G. Pavic, "Measurement of structure borne wave intensity, part I: Formulation of the methods," J. Sound Vib. 49, 221-230 (1976).

16. J. P. Chambard, V. Chalvidant, X. Carniel, and J.-C. Pascal, "Pulsed TV-holography recording for vibration analysis applications," Opt. Lasers Eng. 38, 131-143 (2002).

17. M. P. Petrov, S. I. Stepanov, and A. V. Khomenko, Photorefractive crystals in coherent optical systems, Vol. 59 of Springer Series in Optical Sciences (Springer-Verlag, 1991).

18. J. P. Huignard and A. Marrakchi, "Two-wave mixing and energy transfer in $\mathrm{Bi}_{12} \mathrm{SiO}_{20}$ crystals: application to image amplification and vibration analysis," Opt. Lett. 6, 622-624 (1981).

19. Ph. Delaye, L. A. de Montmorillon, and G. Roosen, "Transmission of time modulated optical signals through an absorbing photorefractive crystal," Opt. Commun. 118, 154164 (1995)

20. J. C. Launay, Institut de Chimie de la Matière Condensée de Bordeaux, Université de Bordeaux I, France (personal communication, 1998).

21. N. A. Korneev and S. I. Stepanov, "Dynamic self-diffraction of laterally vibrating speckle patterns in photorefractive crystals," Optik 91, 61-65 (1992).

22. A. A. Kamshilin, K. Paivasaari, N. I. Nazhestkina, V. V. Prokofiev, S. Ashihara, Y. Lida, T. Shimura, and K. Kuroda, "Adaptive correlation filters for speckle patterns in photorefractive crystals," Appl. Phys. B 68, 1031-1038 (1999). 